\title{
Variations
}

Variations

Revue internationale de théorie critique

11 | 2008

La beauté est dans la rue

\section{Sous le pavé la plage ou la Beauté est dans la rue}

\section{Michal Kozlowski}

\section{(2) OpenEdition \\ Journals}

Édition électronique

URL : http://journals.openedition.org/variations/256

DOI : 10.4000/variations. 256

ISSN : 1968-3960

\section{Éditeur}

Les amis de Variations

\section{Référence électronique}

Michal Kozlowski, « Sous le pavé la plage ou la Beauté est dans la rue », Variations [En ligne], 11 | 2008, mis en ligne le 01 février 2012, consulté le 03 mai 2019. URL : http://journals.openedition.org/ variations/256 ; DOI : 10.4000/variations.256

Ce document a été généré automatiquement le 3 mai 2019.

Les ami•e•s de Variations 


\title{
Sous le pavé la plage ou la Beauté est dans la rue
}

\author{
Michal Kozlowski
}

\section{NOTE DE L'ÉDITEUR}

Première publication sur www.theoriecritique.com, « La beauté est dans la rue ", 1er mai 2008, pp. 18-27

1 Chaque révolte populaire vise la récupération de l'espace commun. Et il ne s'agit pas en premier lieu de «l'espace public» conçu comme un lieu privilégié du pouvoir légitime dans ses nombreuses mutations comme l'espace politique, l'espace culturel ou encore l'espace économique. Bien au contraire, tandis que les rebelles contestent et rejettent le bien-fondé des «espaces publics » - parfois jusqu'à demander leur anéantissement, ils veulent plutôt récupérer l'espace commun. Le premier milieu naturel de la révolte moderne, c'est l'espace urbain. L'espace urbain est ici à la fois une condition de possibilité de l'action collective et son enjeu. "Sous les pavés la plage ", en mai 1968, exprimait aussi cette revendication pour une vie collective meilleure, le droit au plaisir et le droit à la beauté. Certes les pavés servaient ici d'arme, mais la plage incarnait déjà une fin politique. Le mot d'ordre résume une intuition révolutionnaire collective. Mais derrière cette intuition il y avait bel et bien une théorie, actuellement oubliée voir refoulée - celle de Malevitch ou celle des situationnistes, ou encore celle plus ancienne des grandes utopies des Lumières. Quarante ans après la dernière révolution, la question de l'espace commun n'a guère perdu sa pertinence.

Depuis, le système capitaliste mondial a certes connu des transformations importantes, mais il n'a nullement effacé la ville comme milieu privilégié de la production (même si les produits ne sont plus les mêmes), de la reproduction, de la distribution et de l'accumulation. Pour reprendre le terme de Negri, c'est dans les villes que la multitude obtient sa forme la plus intense et la plus paradoxale. C'est ici qu'elle ne cesse de capituler 
devant le capital sans jamais définitivement succomber. La structure spatiale n'est pas contingente par rapport à ce processus, elle semble même jouer un rôle essentiel. Les villes sont nos habitations, nos usines, nos rues, nos ruelles, nos impasses, nos distances et nos connections, nos lieux d'échange et de loisirs, par excellence spatiaux. Il s'agit de quelque chose de moins qu'une architecture (question de goût et de style) mais il s'agit aussi de quelque choses de plus. Nos subjectivités sont non détachables de ces rapports spatiaux. L'espace nous traverse avant qu'on arrive à le traverser. Revenons donc quelques siècles en arrière pour mieux comprendre l'histoire de cet espace.

\section{Espace et modernité}

3 Le père fondateur de notre modernité René Descartes a commencé son investigation sur la nature par la définition classique d'espace. Il l'a d'abord doté de fameuses dimensions : la largeur, la longueur, la profondeur. Puis il l'a considéré comme substance. Cette deuxième considération me semble être la plus intéressante et la plus pesante bien qu'elle ait été ensuite écartée voir renversée. Descartes eut l'intuition splendide de proposer une équation d'emblée choquante : espace égale matière. C'est justement cette matérialité de l'espace que j'essaierai de mettre ici en valeur. La matière-espace n'est ni homogène ni statique. Elle constitue un ensemble dynamique d'agrégats de densité différente, elle est divisible et divisée, structurable et structurée, elle a des forces et des contraintes. La "fabula mundi » cartésienne est une histoire de la transformation multiple mais ordonnée de l'immanence spatiale. La question sur laquelle Descartes nous doit une réponse, c'est celle de savoir d'où vient cette immense dynamique de l'espace-matière? Comment la substance peut-elle avoir une histoire ? Comment arrive la différentiation an sein de l'être par définition infini et éternel ? Et Descartes ne tarde pas à nous donner cette réponse: c'est la puissance divine qui a animé l'espace, c'est sur ordre de l'Être Suprême que le mouvement a commencé. Comme si tout ce qui est spatial obéissait à la volonté. La volonté qui pour sa part ne se représente jamais dans l'espace. On n'en trouve aucune trace particulière - c'est la totalité du mouvement des éléments qui est la seule trace de ce pouvoir primordial. L'espace cartésien est né dans un double mouvement. Le premier consiste dans l'élimination des mouvements qualitatifs dont le moteur sont les éléments métaphysiquement hétérogènes. Cela permet de penser l'espace en termes systémiques. Le deuxième mouvement (jamais définitivement achevé) consiste a détacher la subjectivité et la spatialité (bien que le corps soit entièrement spatial). Le mérite de Descartes réside bien évidement dans ce premier mouvement aussi bien que dans le fait qu'il pressent la nature spatiale du corps vivant aussi bien que le lien occulte entre espace et pouvoir. La spatialité du corps social reconnue dans la renaissance italienne reste pour l'instant négligée...

Descartes vivait encore quand son meilleur et infidèle élève Blaise Pascal a porté le coup mortel à l'intégralité du système cartésien de la nature. Désormais l'équation espacematière ne tient plus car dans l'immanence de la substance conçue comme le «lieu intérieur » se trouve inséré l'hétérogène «lieu extérieur»: le vide. Par conséquent l'espace ne représente guère qu'une potentialité d'avènement de la chose. Le vide comme le "réel néant" ou autrement dit, le néant existant réellement pose une aporie métaphysique insurmontable. Le vide à l'image d'un trou noir commence à absorber l'espace jusqu'à son anéantissement, en tout cas d'un point de vue ontologique. 
D'où probablement le misérable sort que connut l'idée d'espace dans les temps qui suivirent immédiatement. Leibniz lui refusa tout statut ontologique - l'espace leibnizien était de l'ordre épistémologique et encore, de l'ordre de l'épistémologie imparfaite des monades humaines ou animaliers. Il s'agit d'une fiction utile à travers laquelle la connaissance se réalise sous forme d'une approximation vouée à l'efficacité plus qu'à la vérité métaphysique. Le réel ne se constitue que dans l'intériorité de la perception. Le dehors n'est qu'un mirage.

6 Malgré la subtilité de sa démarche, Kant sous certains aspects reste digne héritier de Leibniz. Certes la philosophie critique rejette aussi bien le simple dualisme de Descartes et l'« anti-realisme » spatial de Leibniz. L'espace se trouve à la fois couplé et opposé au temps, comme deux formes d'intuition subjective. Il n'est pas sans importance de remarquer que l'espace, conçu chez Descartes comme lieu intérieur devient ici le «sens extérieur", à la différence de la forme intuitive temporelle dénommée le «sens intérieur ». On revient donc paradoxalement vers le péché originel cartésien. La vie subjective ne se déroule pas en dehors de l'espace mais elle suit une logique du temps. Le "lieu intérieur» est donc extérieur, on peut même dire infiniment extérieur, à la subjectivité.

7 Kant suit cette piste cartésienne à sa manière. Il renvoie l'espace au domaine de la raison théorique - l'ordre de la connaissance. Tout ce qui est spatial est objet de connaissance. En revanche ce qui est pratique, en l'occurrence la subjectivité morale, se constitue en suspendent délibérément toute connaissance possible. L'espace chez Kant, même s'il subsiste dans la médiation constante avec la subjectivité, reste passif, il n'a pas de signification éthique ni d'histoire. Même si Kant reconnaît que nous vivons dans l'espace comme l'espace vit dans nous, il n'arrive pas à thématiser la complexité de cette apparente symétrie. Il est vrai que le rapport entre le sujet et l'espace change dans les jugements du beau et du sublime. Dans ce cadre l'espace effectivement pénètre le sujet en brisant son originaire indifférence morale envers la nature. Le jeu libre des facultés, la tension entre le spatial, le temporel et le téléologique demeure toujours une grande promesse du projet Kantien. Mais cette promesse implicite, Kant et ses disciples semblent l'avoir volontairement écartée.

Hegel médiatise la subjectivité et l'espace politiquement et historiquement. L'espacenature est le premier objet de la négation, il fait donc émerger la subjectivité mais établit aussi l'horizon ultime de cette subjectivité - la mort individuelle. Le premier stade de la subjectivité consiste à dominer l'espace, le dernier à y trouver sa sépulture. Hegel conçoit l'espace comme une forme radicale d'aliénation de la substance. Il n'y a que l'espace éternel qui soit véritablement «en soi » sans être "pour soi ». L'idée que l'espace soit extérieur par rapport à la subjectivité n'est donc pas dépassée. Hegel trace dans l'histoire universelle le passage dialectique de l'espace vers le temps. L'affinité avec Kant est plus forte qu'on pourrait le soupçonner.

Hegel inaugure en quelque sorte la priorité épistémique octroyée au temporel. Chez lui comme chez Marx le temporel trouve son essence dans l'histoire. L'histoire fournit une médiatisation entre le spatial et le temporel. A leur suite montent en force des démarches qui absolutisent la temporalité. Heidegger, qui pèse sur les sciences humaines de notre temps, en témoigne d'une manière suggestive. Il conçoit le temporel comme le Devenir fondamental qui précède toute forme possible du spatial. Par conséquent l'espace est réduit à un simple "lieu d'émergence » du signifiant. L'espace en tant que le milieu du vécu subjectif, subit de nouveaux une sorte de transcendantalisation. Il n'est plus 
substance, il n'est plus matériel - il n'est qu'une condition de possibilité. On peut se risquer à dire que depuis Descartes on a affaire avec un processus continu de transcendantalisation de l'espace. Chez Kant et Leibniz, cette transcendentalisation est explicite. Mais chez Hegel, l'espace n'est guère plus que une condition de possibilité de la subjectivité, une condition en soi sans signification, qui se trouve historiquement dépassée dans l'union finale entre la liberté et la raison. Dorénavant la modernité produit une multiplicité d'espaces transcendantaux et abstraits qui ne sont pas pour autant illusoires ni fictionnels. Mais leur enracinement dans le réel est toujours emprunté à l'espace tridimensionnel des corps vivants. Dans ce sens on peut oser dire que ces nouveaux espaces restent otages de l'espace cartésien.

10 Nous ne plaidons évidement pas pour le renversement des acquis théoriques de l'épistémologie moderne et encore moins des découvertes la physique contemporaine. Si la réflexion sur la substantialité de l'espace nous semble être pertinente, c'est dans le domaine du social et du politique. Or il semble évidant que le caractère profondément politique de l'espace n'a jamais été négligé par les pouvoirs, et a très souvent été sousestimé par les philosophes et les idéologues. C'est dans ce sens qu'il faut revenir à Descartes. Il faut oser penser l'espace non comme une abstraction mais comme un concret, non comme un vide mais comme une matière et finalement, non en termes de négativité mais comme une positivité qui engendre le pouvoir. Selon une expression de Deleuze empruntée à Spinoza, exercer le pouvoir c'est agir sur l'agir. Agir sur l'agir veut dire produire les sujets, faire la subjectivité. Or précisément l'espace produit les sujets dans le sens positif, non comme lieu où les sujets se déploient mais comme une machine qui fait et à la fois fait faire. Le modèle cartésien, indépendamment de sa place au sein du système métaphysique, peut se montrer ici très utile. À l'exception de la glande pinéale, Descartes détache presque totalement le cogito de l'espace-matière. Nous proposons le contraire : il faut penser le sujet comme indissociable de l'espace. Indissociable veut dire non seulement connecté mais qui fait partie du même système. Il faut donc dire que le sujet est matériel, que le sujet est spatial. Mais il ne s'agit pas ici de revenir au "matérialisme » natif qui essaie d'attribuer toutes les caractéristiques «objectives» de l'espace à la subjectivité. Si le sujet est spatial, l'espace, l'espace social au moins, est subjectif. Et encore, il n'est pas subjectif parce que relatif aux sujets. Il est subjectif parce qu'il comporte des traces subjectives, parce qu'il est non seulement désirable mais aussi désirant, non seulement répugné mais aussi répugnant.

\section{L'espace transcendantal capitaliste}

11 L'idée même d'espace transcendantal qui a ses origines chez Kant et Leibniz présuppose que l'espace sera otage de la subjectivité. Non dans un sens où il serait une simple projection de chaque sujet empirique, mais dans un sens universel et génétique. La relation entre les deux n'est donc jamais symétrique, elle est plutôt dialectique, même s'il faut admettre que ce terme reste vague. Depuis un siècle et demi environ, on a vu l'avènement de plusieurs formes nouvelles de l'espace transcendantal. Le premier et le plus important, c'est sans doute le marché d'Adam Smith et David Ricardo. Le concept de marché, comme nous le savons, provient de la transformation d'un terme décrivant une simple réalité spatiale, en occurrence la bonne vielle place du marché, devenue de nos jours un lieu de tourisme et de loisirs sans grande importance. Géographiquement au centre-ville - en réalité déjà dans les marges. Le marché d'autrefois était un endroit 
physique et spatial organisé en carré, ou bien en Orient une via recta que nous connaissons aujourd'hui sous le nom arabe de souk. Le marché était un lieu d'échange de biens. L'échange présupposait l'équivalence de valeur des biens échangés. Cette possibilité d'équivalence était aussi assurée par la forme spatiale - la proximité des parties, présence immédiate des marchandises et finalement le caractère public de la place.

Avant que n'apparaisse le marché capitaliste comme marché transcendantal, avec sa subjectivité transcendantale comme subjectivité du capitaliste, l'espace doit connaître plusieurs transformations, regroupements, démontages, construction de nouveaux sousespaces et connections entre eux. Marx décrit ce processus dans le premier livre du Capital dans le chapitre XXIV consacré à l'accumulation primitive. Même si Marx ne se focalise pas sur le problème de l'espace, on se rappelle bien que la première étape de l'accumulation passe par le «fencing » et le «clearing of estates ». Et il ne s'agissait pas ici seulement de priver le peuple d'un espace nourricier. Ce processus consiste en premier lieu en une nouvelle délimitation de la structure spatiale de la société, afin de gouverner les mouvements des corps vivants. Bien que la clôture soit importante, ce qui est essentiel dans cette révolution spatiale, ce sont précisément les chemins de fuite ${ }^{1}$. Transformer les paysans en vagabonds immédiatement confrontés à des lois sévères contre le vagabondage fut la première étape. Mais quel rapport à l'espace implique le vagabondage? Le vagabond est quelqu'un qui circule librement sur les routes publiques. Il s'agit de la condition nomade - le mouvement du corps flottant en permanence, détaché du lieu du domicile et de celui du travail.

Il nous semble qu'il existe une dimension cruciale de ce processus capitaliste, à peine thématisée par Marx ${ }^{2}$ mais en revanche très bien saisie par Foucault, surtout dans Surveiller et Punir mais aussi dans ses cours au Collège de France de 1977-1978, « Sécurité, Territoire, Population». La subjectivité vagabonde doit être dépassée et l'espace vagabond aboli. Cela par le travail disciplinaire. Foucault analyse le régime disciplinaire - jusqu'au régime panoptique avant tout comme un régime spatial. Le régime disciplinaire représente un stade intermédiaire entre régime du simple échange et régime d'accumulation capitaliste comme accumulation transcendantale.

Le système correctionnel n'est plus un symbole démonstratif - il doit devenir un moyen efficace de gestion de la société. Cela implique une procédure suffisamment simple pour pouvoir être appliquée au grand nombre de détenus. L'autre exigence est que le détenu doit être transformé d'une nouvelle manière - il ne s'agit plus seulement de lui faire intérioriser telle ou telle norme, mais de former en lui une disposition permanente à l'intériorisation. La mécanique du pouvoir doit passer par une double épreuve d'efficacité. Elle doit tout d'abord discipliner les corps pour les investir ensuite d'une capacité à se discipliner eux-mêmes. Cette deuxième épreuve renvoie déjà d'une manière directe à la subjectivité. Le pouvoir disciplinaire, qui correspond historiquement au développement du régime libéral, a aussi un nouvel objectif politique : au lieu d'affirmer la puissance du pouvoir dans le cadre du conflit avec le peuple, il doit éliminer ce conflit. Cela doit se produire dans le cadre d'une politique rousseauiste renversée. Au lieu que le peuple s'approprie le pouvoir, c'est le pouvoir qui confisque le peuple. Mais l'effet d'intégration y est accompli.

En tous cas, le pouvoir commence par le corps : « [il s'agit] d'exercer sur lui une correction tenue, d'assurer des prises au niveau même de la mécanique - mouvements, gestes, attitudes, rapidité : pouvoir infinitésimal sur le corps actif. ${ }^{3}$ ” Avant, la seule réceptivité qui intéressait le 
pouvoir c'était la capacité de ressentir la souffrance ; maintenant, c'est une autre qualité qui attire l'attention : le corps est modifiable dans son activité. Non seulement il peut être mutilé et massacré, mais il peut aussi agir selon la règle qu'on lui impose. Cette idée est fondée d'une part sur des théories mécanistes du corps comme celles de Descartes ou de La Mettrie - sur le savoir très spécifique qui se déploie au cours du XVIII siècle, et de l'autre elle est une nécessité technologique d'efficacité. C'est l'époque où les institutions les plus performantes sont les armées. Pourquoi alors ne pas modeler les autres collectivités sur cet exemple?

En fait, la manière dont le pouvoir se figure le corps porte encore beaucoup de traces du cartésianisme. Or le sujet (âme) qui habite le corps n'importe plus. Si les comportements du corps peuvent être normalisés et contrôlés à travers une simple opération mécanique, pourquoi faire appel au sujet? «La contrainte porte sur les forces plutôt que sur les signes; la seule cérémonie qui importe vraiment, c'est celle de l'exercice. $»^{4}$ Le pouvoir ne cherche plus à se manifester - il veut s'exercer. Mais ce n'est que le début. Le cartésianisme du système correctionnel est pour le moins ambigu. Fondé sur la découverte qu'il n'est pas nécessaire de s'adresser au sujet pour transformer le corps, il ne se contentera pas de cette constatation. Le nouveau savoir sur le sujet que la discipline prépare, c'est que si le sujet existe, il est fait du corps, que la corporéité est sa seule matière.

La répartition de l'espace et du temps, cette nouvelle architecture de la coercition n'atteint d'abord qu'un effet d'habitus. Le corps-machine ou plutôt les grands ensembles des corps-machines doivent agir automatiquement. Dans le règlement de la Maison de jeunes détenues à Paris, nous trouvons l'article 19 qui prévoit que «la prière est faite par l'aumônier d'une lecture morale ou religieuse. Cet exercice ne doit pas durer plus d'une demi heure. ${ }^{5}$ La persuasion ou l'endoctrinement sont toujours présents mais leur rôle est secondaire. Les architectes sociaux du XIXe n'osent pas les abandonner, peut-être par pudeur, mais ils n'y attachent pas trop d'importance. Il est impossible « d'approfondir » le corps et de lui fournir des mécanismes d'auto-contrôle ou d'auto modification. Avant tout il est impossible de lui faire aimer le pouvoir.

Le système correctionnel constitue donc un moyen parfait pour la fabrication de certaines catégories d'individus. Les soldats et les ouvriers notamment sont indispensables pour mettre en œuvre une nouvelle société avec ses immenses projets. $\mathrm{Si}$ le cartésianisme invente le monde comme gérable et radicalement transformable, la seule question qui émerge dans cette logique est la question des outils. Les grosses collectivités d'individus normalisés et disciplinés offrent la réponse : elles sont des instruments pour la transformation de la nature. Mais quelle nature doivent-elles transformer? Dans l'opposition entre l'homme et la nature, aucun enjeu pratique n'explique d'une manière satisfaisante le fonctionnement du pouvoir. L'enjeu du pouvoir est toujours défini dans les rapports à l'intérieur de la société. Les hommes ne sont donc jamais uniquement des outils, ils constituent aussi des fins. C'est une transformation macabre de l'impératif kantien. Le pouvoir se représente la société comme son objet. Et le pouvoir est indissociable de l'espace. Les grands édifices architecturaux de notre civilisation, les prisons, les usines mais aussi les armées comme les constructions corporelles mobiles semblent aujourd'hui être marginalisés mais leur disparition n'est pas à l'ordre du jour. Ils constituent toujours le noyau dur et le dernier rempart de l'ordre social actuel. Parfois les formes nouvelles du gouvernement ne sont qu'apparemment en conflit avec les anciennes. Pour le moment il nous suffit de dire que cette formidable machinerie spatiale 
n'est là que pour produire l'espace systémique du capitalisme mûr. Le vieux marché ne peut se faire remplacer par le marché capitaliste qu'à travers l'abstraction de l'échange équivalent, de la valeur d'usage et du lieu déterminé. Autrement dit, il faut effacer le caractère spatial et matériel de la valeur.

Faire abstraction de l'échange des valeurs et du coté matériel de la circulation veut dire aussi mettre à part l'espace cartésien au profit de l'argent sous forme de capital. Le capital lui, trouve son origine dans le domaine de la production. La production quant à elle est une affaire de plus-value. Le jeu abstrait est en fait le jeu transcendantal qui subordonne l'espace à un espace sans dimensions - le réseau qui fait faire l'accumulation. L'argent n'a pas d'utilité ni d'épaisseur. Le processus d'accumulation est né dans l'espace pour en sortir. Le marché économique moderne digère l'espace pour accumuler.

\section{Contre la séparation}

20 Le capital représente effectivement une forme politiquement primaire de l'espace transcendantal dirigeant voir dominateur. Un monde comme le notre connait une multiplicité d'espaces transcendantaux et on en ajoute toujours plus. Il y a l'espace politique, l'espace médiatique, l'espace économique et social - bien distinct de l'espace financier (dont les limites et les connexions internes se détachent de plus en plus des lieux du travail et de l'échange). Un espace en pleine expansion depuis quelques décennies, l'espace informationnel. Mais toutes ces formes d'espace ne sont-elles pas parasitaires par rapport au simple espace vital commun qui co-constitue notre corporéité ? Quels effets produisent ces entités idéales sur la « réalité spatiale »? On parle beaucoup de délocalisation, d'extériorisation, de militarisation de frontières, de ghettoïsation, de monde en réseau... On peut risquer un terme plus général pour décrire cette série de phénomènes - il s'agit d'effet de séparation. Si les vieux édifices modernes de la gouvernementalité s'exerçaient à travers l'agrégation d'éléments hétérogènes et chaotiques, les forces nouvelles poussent à séparer les agrégats. La séparation disperse, diffuse et apporte le chaos, mais elle le fait d'une manière systémique et ordonnée. Mais l'effet de la séparation reste toujours celui de l'aliénation. Cette fois, on n'a plus besoin de recourir à un essentialisme quelconque. Il n'est pas nécessaire de faire la scolastique de la nature (de l'homme) et sa forme historiquement contingente. Après tout, la séparation veut dire la mise en distance en espace, ou l'institution d'un blocus.

21 L'espace fabuleux d'Internet produit des centaines de millions des corps assis, penchés devant les machines numériques. L'entreprise moderne n'a plus besoin de la proximité des travailleurs. Les ingénieurs sont sortis des usines pour s'installer dans des bureaux spécialisés. Les ouvriers, eux aussi, se trouvent " hors usine », ils sont ailleurs, dans les plus ou moins petites entreprises sous-traitantes. Les managers n'ont plus aucun lien avec l'usine, d'ailleurs ils sont constamment recyclés et ne prennent plus les responsabilités décisives. La gestion stratégique se fait via des firmes de consulting ou encore des private equity funds. Les riches n'acceptent plus d'habiter les mêmes immeubles ou quartiers que les pauvres. Leurs enfants ne se fréquentent plus. Les rapports de classe disparaissent tout simplement parce que les classes n'entrent plus en aucune relation. L'Amérique et l'Europe à l'image d'Israël se transforment en réseaux de forteresses. D'ailleurs, les récents succès commerciaux dans le domaine des nouvelles technologies en provenance de l'État hébreux sont particulièrement parlants: ils sont dû en grande partie aux technologies de la sécurité. L'industrie la plus rentable de notre temps c'est celle de la 
sécurité, la vidéo-surveillance s'impose de plus en plus dans la structure urbaine. Les nouveaux centres commerciaux réalisent de manière macabre une vision leibnizienne d'un monde sans espace. Comme les monades il n'ont pas de fenêtres, le réel se trouve à l'intérieur sans extériorité possible. L'espace autour d'un centre commercial n'est plus que le néant urbain privé de toute signification. C'est pour cela qu'aujourd'hui plus que jamais, il est essentiel de lutter contre l'aménagement capitaliste de l'espace. Tout se passe comme si le grand processus intégrateur (au niveau transcendantal) de la mondialisation passait par de multiples et toujours locales (donc réelles) microopérations de partage, de division et de séparation. La lutte anticapitaliste ne peut plus se permettre le confort de faire abstraction de cette réalité primordiale. Le capitalisme se nourrit de notre espace, il faut le mettre au régime.

\section{NOTES}

1. Il faut remarquer ici que dans le mouvement spatial de la transformation sociale il n'existe guère de fermetures définitives. Les fermetures sont toujours relatives, afin de provoquer le mouvement soit du renversement soit de la reproduction de l'ordre social. La seule exception est peut-être le camp de concentration nazi - à juste titre considéré par certains comme la vraie fin d'histoire.

2. Mais il existe une formidable tradition marxiste dans le champ des études urbaines qui problématise la question de l'espace. Je pense notamment à Henri Lefèbvre et son ouvrage La Production de l'espace, mais aussi à David Harvey avec Spaces of Capital: Towards a Critical Geography.

3. M. Foucault, „Surveiller et Punir”, Gallimard 1975, p. 139

4. Ibidem. p. 139

5. Ibidem p. 12

\section{AUTEUR}

\section{MICHAL KOZLOWSKI}

Maître de conférences en philosophie contemporaine, Université de Varsovie 